\title{
SEGMENTATION OF 4D MR RENOGRAPHY IMAGES USING TEMPORAL DYNAMICS IN A LEVEL SET FRAMEWORK
}

\author{
Ting Song ${ }^{1,2}$, Vivian S. Lee ${ }^{2}$, Henry Rusinek ${ }^{2}$, Qun Chen ${ }^{2}$, Louisa Bokacheva ${ }^{2}$, Andrew Laine ${ }^{1}$ \\ ${ }^{1}$ Department of Biomedical Engineering, Columbia University, New York, NY, USA \\ ${ }^{2}$ Department of Radiology, New York University School of Medicine, New York, NY, USA
}

\begin{abstract}
A novel 4D level set framework was developed to segment dynamic MR images into the cortex, medulla and collecting system. The novelty of the method is that it combines information from spatial anatomical structures and temporal dynamics. The accuracy of the fully automatic 4D level set algorithm was found to be comparable to manual segmentation performed by experts on renal anatomy. The algorithm requires less than one minute to automatically segment a single kidney $4 \mathrm{D}$ patient data set with more than 40 time points.
\end{abstract}

Index Terms - MR renography, dynamic contrast enhanced MRI, image segmentation, level set, temporal dynamics

\section{INTRODUCTION}

Segmentation of dynamic MR images of the kidney into the cortex, medulla and collecting system plays a key role in renal function measurements using multicompartment models [1]. The challenge in segmenting dynamic contrast enhanced images is that when contrast agent wash-in and wash-out occurs, image intensity values change rapidly as the time series evolves. Poor kidney function or stenotic vasculature may prevent the uptake of contrast agent, resulting in disjointed demarcation of kidney structures. Accurate and continuous boundary delineation is not always feasible. Moreover, the contrast agent can also wash into neighboring tissues, such as the spleen and liver.

Existing kidney segmentation techniques can be divided in two basic categories: spatial and temporal. Traditional segmentation is performed in the spatial domain. Especially when the contrast provides good corticomedullary differentiation, the operator segments serially first the entire kidney, then the cortex and medulla [2-4]. Temporal or vector segmentation considers each voxel's intensity time course as a vector and classifies the tissues according to their distinct features and behaviors in the temporal domain [5-8]. The temporal pattern of contrast uptake is a strong feature to distinguish kidney tissues from surrounding tissues as well as to differentiate various intrarenal tissues. Due to differences in vasculature, filtration, and re-absorption, different tissues of interest are enhanced during different phases of the acquisition. For example, in a normal kidney, the peak uptake in the cortex occurs approximately 30 seconds after injection, in the medulla at 2-3 minutes, and in the collecting system at 4-6 minutes.

The advantage of the spatial segmentation approach is that it fully utilizes the contrast across anatomical structures of kidney tissues. Segmentation of 3D MRR can be achieved by many approaches, including optimized thresholding, graph cuts, and 3D level set. The advantage of the temporal segmentation method is that it obtains time information across all of the data; however, it ignores the spatial anatomical structure. Therefore, the purpose of the present study is to combine both spatial and temporal information using a novel temporal dynamics 4D level set segmentation approach.

\section{METHODOLOGY}

\subsection{Spatial 3D Automated Seed Detection}

In order to efficiently apply temporal dynamics in a 4D level set on MR renography (MRR), a 3D automated initialization or seeds detection is used. In this paper, a 3D spatial level set segmentation method, which perform a minimal partitioning of the image data into piecewise constant objects based on Mumford-Shah functional, was used due to its flexibility and efficiency. After this step, a rough segmentation, or auto-detected seeds, of each renal tissue (cortex, medulla, and collecting system) are extracted. These seeds are then used as the automatic initialization for the next step.

\subsection{Temporal Dynamics 4D Level Set}

This method utilizes temporal dynamics as vector inputs. $\overrightarrow{\mathbf{I}}(\overrightarrow{\mathbf{x}}, t)$ is a $4 \mathrm{D}$ image data set. The spatial domain $\Omega=\Omega(\overrightarrow{\mathbf{x}})$ defines 3D space where the kidney resides. Each voxel in the image $\overrightarrow{\mathbf{I}}(\overrightarrow{\mathbf{x}}, t)$ is defined by spatial coordinates $\overrightarrow{\mathbf{x}}$ and a temporal dynamics defined by the temporal variable $t$. These types of images differ from the traditional 2D plus time or 3D plus time images such as cine MR [9], where only motion information contained in the temporal dimension and the properties such as intensity of corresponding voxels do not change over time. On the contrary, 4D images with temporal dynamics such as 4D 
renography usually reflect both motion and changes in the voxel properties, which increases the difficulty of segmentation. To simplify the analysis, our study assumes that $\overrightarrow{\mathbf{I}}(\overrightarrow{\mathbf{x}}, t)$ has been registered, i.e., for each voxel $\overrightarrow{\mathbf{x}}$ in the spatial domain the vector $\overrightarrow{\mathbf{u}}(\overrightarrow{\mathbf{x}})$ represents the dynamic activity of single renal tissue.

With these settings, a framework of temporal dynamics 4D level set is proposed as follows. Given a 4D image data set $\overrightarrow{\mathbf{I}}(\overrightarrow{\mathbf{x}}, t)$ with temporal dynamics defining the spatial domain $\Omega=\Omega(\overrightarrow{\mathbf{x}})$, a $4 \mathrm{D}$ deformable model utilizing temporal vector $\overrightarrow{\mathbf{u}}(\overrightarrow{\mathbf{x}})$ with associated segmentation energy is used to separate the image into objects and background. A homogeneity-based energy functional is adapted to segment piecewise constant or piecewise smooth 4D dynamics with each partition of the images. Assuming an object represented by temporal dynamics vector $\overrightarrow{\mathbf{c}_{\mathbf{1}}}$, and a background represented by dynamics vector $\overrightarrow{\mathbf{c}_{2}}$, separated by the surface $C$, the proposed energy functional is defined as:

$$
\begin{aligned}
F\left(C, \overrightarrow{\mathbf{c}_{1}}, \overrightarrow{\mathbf{c}_{2}}\right)= & \mu(\text { SurfaceArea }(C)) \\
& +\lambda_{1} \int_{\text {inside }(C)}\left(\overrightarrow{\mathbf{u}}-\overrightarrow{\mathbf{c}_{1}}\right)^{\mathbf{T}}\left(\overrightarrow{\mathbf{u}}-\overrightarrow{\mathbf{c}_{1}}\right) d \Omega, \\
& +\lambda_{2} \int_{\text {outside }(C)}\left(\overrightarrow{\mathbf{u}}-\overrightarrow{\mathbf{c}_{2}}\right)^{\mathbf{T}}\left(\overrightarrow{\mathbf{u}}-\overrightarrow{\mathbf{c}_{2}}\right) d \Omega
\end{aligned}
$$

where $\mu \geq 0, \lambda_{1}, \lambda_{2} \geq 0$ are fixed parameters.

Segmentation of the data is performed via minimization of the functional $F$ with respect to $\left(C, \overrightarrow{\mathbf{c}_{1}}, \overrightarrow{\mathbf{c}_{2}}\right)$. The energy minimization can be performed under a traditional level set framework [10]:

$$
\begin{aligned}
\frac{\partial \phi(\overrightarrow{\mathbf{x}})}{\partial t} & =\mu \operatorname{div}\left(\frac{\nabla \phi(\overrightarrow{\mathbf{x}})}{|\nabla \phi(\overrightarrow{\mathbf{x}})|}\right)+\lambda_{1}\left(\overrightarrow{\mathbf{u}}(\overrightarrow{\mathbf{x}})-\overrightarrow{\mathbf{c}_{1}}\right)^{\mathbf{T}}\left(\overrightarrow{\mathbf{u}}(\overrightarrow{\mathbf{x}})-\overrightarrow{\mathbf{c}_{1}}\right) \\
& -\lambda_{2}\left(\overrightarrow{\mathbf{u}}(\overrightarrow{\mathbf{x}})-\overrightarrow{\mathbf{c}_{2}}\right)^{\mathbf{T}}\left(\overrightarrow{\mathbf{u}}(\overrightarrow{\mathbf{x}})-\overrightarrow{\mathbf{c}_{2}}\right)
\end{aligned}
$$

where $\phi(\overrightarrow{\mathbf{x}})$ is the level function.

\subsection{Implementation Optimization}

The improvements in the proposed method solve the instability and complexity of numerical implementation. Since the temporal dynamic 4D level set method is dealing with a very large 4D data set, close to one gigabyte size, it is important to minimize the complexity of the numerical scheme. An iterative numerical approach was applied in this study.

Our 4D framework takes more time for convergence in each iteration as well as across all iterations compared to a $3 \mathrm{D}$ version. For this reason, both accuracy and efficiency must be considered during implementation. Otherwise, the method would be prone to numerical instabilities and would be clinically impractical due to the long time required for segmenting each 4D data set.

Equation (2) is composed of two terms: a curvature term and a homogeneity term. Special consideration for each term was applied during the actual numerical implementation.

The first term on the right-hand side of equation (2), $\mu \operatorname{div}(\nabla \phi(\overrightarrow{\mathbf{x}}) /|\nabla \phi(\overrightarrow{\mathbf{x}})|)$, serves as a curvature term that controls the spatial smoothness of the surface $C$. This term involves a numerical differentiation of the level set function. In the original implementation [11], an "upwind" implementation scheme was proposed, involving computing forward and backward difference and a rather complicated differentiation scheme. If the sign of the current level set function at each pixel is used as a signed distance function, in general, it is not differentiable at every point. (The exception is provided by the case of a planar boundary.) In most medical imaging applications,, large numerical errors may occur at those singular points introduced by numerical differentiation and propagate to neighboring pixels during each iteration, causing numerical instability.

Although this problem may be partially be solved by reinitializing of the level set function after several iterations, re-initialization may introduce new problems as well, especially when segmenting a convoluted surface such as the kidney medulla. Re-initialization is time-consuming and it discards all updates to the level set function at the current iteration. For this reason, re-initialization is usually performed less frequently, e.g. every 10 iterations, in order to give enough time for accumulating the updates from the dynamic equation(2). However, for a convoluted surface like the kidney medulla, singular points occur close to the boundary, requiring a frequent re-initializations, about every 1-2 iterations, since large errors generated at those singular points will quickly affect the boundary.

In segmentations involving convoluted surfaces, the curvature term will introduce problems caused by numerical differentiation. In this study, targeting kidney segmentation, an alternative implementation for the curvature term or the smoothness constraint was used to avoid numerical differentiation and unnecessary re-initialization caused by it. Given the level set function $\phi$ is a signed distance function, $|\nabla \phi(\overrightarrow{\mathbf{x}})|=1$. Then part of the equation (2) can be reduced to:

$\frac{\partial \phi(\overrightarrow{\mathbf{x}})}{\partial t} \approx \mu \operatorname{div}(\nabla \phi(\overrightarrow{\mathbf{x}}))$,

which is a approximation of a diffusion process. The analytical solution of equation (3) is a Gaussian convolution of the level set function $\phi$, which includes an efficient implementation for $\mathrm{N}$-D filtering and does not require taking derivatives of $\phi$. For this reason, in the actual 
implementation, the curvature term is replaced by the corresponding weighted corresponding updates generated by Gaussian filtering. The force will be balanced with homogeneity term to do the segmentation in each iteration.

The second part of equation (2), $\lambda_{1}\left(\overrightarrow{\mathbf{u}}(\overrightarrow{\mathbf{x}})-\overrightarrow{\mathbf{c}_{1}}\right)^{\mathrm{T}}\left(\overrightarrow{\mathbf{u}}(\overrightarrow{\mathbf{x}})-\overrightarrow{\mathbf{c}_{1}}\right)-\lambda_{2}\left(\overrightarrow{\mathbf{u}}(\overrightarrow{\mathbf{x}})-\overrightarrow{\mathbf{c}_{2}}\right)^{\mathrm{T}}\left(\overrightarrow{\mathbf{u}}(\overrightarrow{\mathbf{x}})-\overrightarrow{\mathbf{c}_{2}}\right)$, is the main driving force of the $4 \mathrm{D}$ segmentation. The temporal dynamics for each pixel can be set as the signal intensity changes over time. However,. 4D MR image segmentation that involves large fields of view must deal with the problem of signal inhomogeneity across slices. This problem is significantly more pronounced than in segmentation of $2 \mathrm{D}$ or $2 \mathrm{D}$ plus time MR [8]. These nonuniformities lead to different starting points, mean values, and any other properties involving intensities. Moreover, differences occur with segmentations that are based only on scalar pixel values, not the temporal dynamics, because the energy of the temporal dynamics in the $4 \mathrm{D}$ level set also affect the homogeneity measurements and bias the 4D segmentation. For this reason, the mean temporal dynamics of each pixel were removed, followed by a normalization based on the total energy of each resulting time course. This way the temporal dynamic 4D level set is free from the inhomogeneity in the MR images, and does not depend on the factors such as injection concentration that affect the energy of the temporal dynamics. Another benefit of using a normalized time course is the increased numerical stability.

\subsection{Clinical Patient's Data Acquisition}

Dynamic MRR images were acquired on a $1.5 \mathrm{~T}$ system (Avanto; Siemens, Erlangen, Germany). 3D T1-weighted spoiled gradient echo imaging was performed in the oblique coronal orientation to include the abdominal aorta and both kidneys with TR/TE/flip angle 2.8/1.1/12 ${ }^{\circ}$; matrix was 161 $\times 256 \times 20$, FOV was $425 \times 425 \times 100 \mathrm{~mm}$; voxel size $1.6 \times$ $1.6 \times 2.5 \mathrm{~mm}$ after interpolation; bandwidth $650 \mathrm{~Hz} /$ voxel; and temporal resolution $3 \mathrm{sec}$. A $4 \mathrm{ml}$ bolus of Gd-DTPA (Magnevist; Berlex Laboratories, Wayne, NJ, USA) was injected after five pre-contrast image acquisitions, followed by $20 \mathrm{ml}$ of saline at $2 \mathrm{ml} / \mathrm{sec}$. Over $20 \mathrm{~min}, 36$ post-contrast images were acquired. For image processing, all $413 \mathrm{D}$ data sets were analyzed using proposed method. Cortex, medulla and collecting system were segmented and compared with two reader's manual tracing results. Two experienced individuals collaborated to manually segment each of the 22 kidneys into cortical, medullary and collecting system regions. These observers used an interactive, locally developed Unix-based software package that provides the user with the ability to view and scroll through the 3D data and construct volumes of interest in arbitrary planes. The manual segmentation took on the average of 2.5 hours per kidney.

\subsection{Validation Analysis}

Manually labeled 3D ground truth data were performed by two experienced radiologists. There were a total of 22 kidneys that had renovascular disease. Manual segmentation took approximately 2.5 hours per kidney. The proposed method included 3D automated seed detection and the automated temporal dynamics 4D level set only took approximately 1 min per kidney.

\subsubsection{Volume Evaluation}

The volumes of automatically segmented and manually segmented results were compared for each cortex, medulla and collecting system. Errors between computer and human ( $V_{\text {error } 1)}$ were compared to errors obtained from two separate manual segmentations ( $V_{\text {error } 2}$ ).

$$
\begin{aligned}
& V_{\text {error } 1}=\left(\left|V_{\text {seg }}-V_{\text {user } 1}\right|+\left|V_{\text {seg }}-V_{\text {user } 2}\right|\right) / 2 . \\
& V_{\text {error } 2}=\left|V_{\text {user } 1}-V_{\text {user } 2}\right| .
\end{aligned}
$$

$V_{\text {error1 }}$ is a measure of the segmentation accuracy for the proposed method. Verror2 is an estimation of interobserver disagreement.

\subsubsection{Signal Intensity (SI) Time Curve Evaluation}

In kidney diagnoses, accurate measurements of signal intensity (SI) curves are directly related to the kinetic model calculation results, which give estimations of important physiological parameters for diagnoses. For this reason, SI curves were also used in the validation study. SI curves for each compartment of the kidney that were estimated from automated segmentation were also compared to the gold standard curves using manual segmentation. Similar to the validation based on volume measurement, $S I_{\text {error1 }}$ was compared with $S I_{\text {error2 }}$.

$$
\begin{aligned}
& S I_{\text {error } 1}=\left(\begin{array}{l}
\left|S I_{\text {Seg }}-S I_{\text {user } 1}\right| / S I_{\text {user } 1} \\
+\left|S I_{\text {seg }}-S I_{\text {user } 2}\right| / S I_{\text {user } 2}
\end{array}\right) / 2 \text {. } \\
& S I_{\text {error } 2}=\left|S I_{\text {user } 2}-S I_{\text {user } 1}\right| / S I_{\text {user } 1} \text {. }
\end{aligned}
$$

\section{RESULTS}

\subsection{Volume Evaluation}

Across 22 kidneys, the volume measurement showed that the discrepancy between the $4 \mathrm{D}$ level set method and corresponding segmentation by expert radiologists was 12.6 $\pm 8.6 \mathrm{ml}, 10.6 \pm 4.9 \mathrm{ml}$, and $2.9 \pm 2.1 \mathrm{ml}$ respectively for the cortex, medulla and collecting system. The discrepancy between two experts was $16.3 \pm 11.2 \mathrm{ml}, 15.9 \pm 5.7 \mathrm{ml}$, and $2.6 \pm 1.7 \mathrm{ml}$. An example of a typical segmented cortex, medulla and collecting system is shown in Fig. 1. 


\subsection{Signal Intensity (SI) Time Curve Evaluation}

For average signal intensity versus time across all kidneys, there was consistently good agreement between enhancement curves derived from the two manually segmentations and the 4D level set segmentation. The mean absolute relative errors were $5.3 \% \pm 2.2 \%$, $4.6 \% \pm 3.5 \%$, and $3.8 \% \pm 2.9 \%$ respectively for the cortex, medulla and collecting system. By comparison, the errors between two experts were $5.9 \% \pm 2.3 \%, 4.7 \% \pm 2.3 \%$, and $5.6 \% \pm 5.4$ $\%$ correspondingly. The t-test results suggested that the volume errors and SI errors of the proposed segmentation method compared to an expert were statistically comparable to the inter-observer variability for all three renal structures.

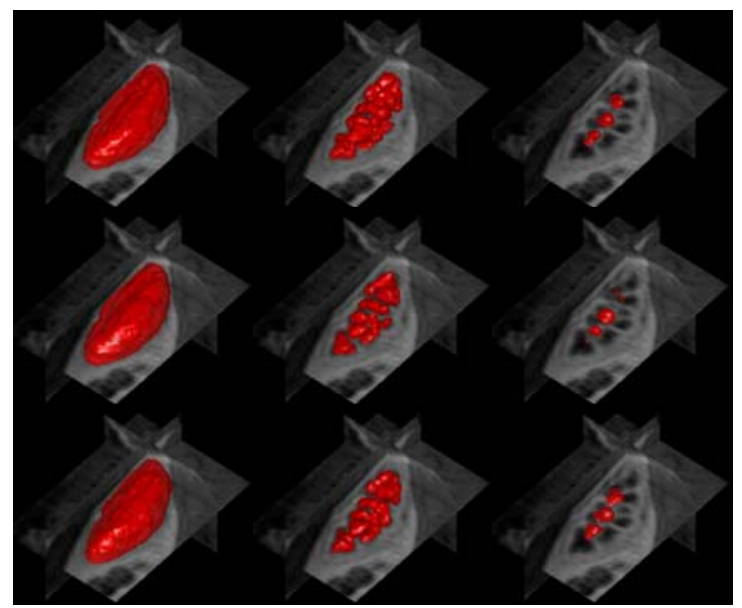

Fig. 1: 3D segmentation achieved by two experts (first two rows) and our algorithm (3rd row); cortex, medulla, and collecting system are shown in 1st, 2nd, and 3rd columns.

\section{CONCLUSIONS}

In 4D (3D plus time) MRI renography, manual delineation of each 4D dataset typically requires approximately 2.5 hours of a radiologist time at a workstation per case. This remains prohibitively costly and labor-intensive for practical clinical use. There are very few methods have been reported for segmenting 4D renography. The existing methods were based on purely spatial segmentation of 2-4 frames out of the entire data set, ignoring the wealth of the temporal dynamics information contained in the data sets. In this context, a novel 4D segmentation framework based on a temporal dynamic 4D level set was proposed. Additional optimization approaches were used in the numerical implementation to increase the efficiency, accuracy, and the numerical stability. The proposed method required less than 1 minute to automatically segment a 4D data set with more than 40 time points. The proposed framework utilized an automated seed extraction mechanism based on a 3D level set to avoid manual initialization used in other existing methods. The novelty of the method is that it combines both information from spatial anatomical structures and temporal dynamics from the time axis. Furthermore, an accurate and simplified substitution of a mean curvature term increased the speed of the 4D level set by at least ten-fold. Compared to manually segmented results. Both volume and signal intensity errors were comparable to the range of inter-observer variance between experienced radiologists. For future studies, statistical analysis will be helpful to determine the performance in terms of statistically significance.

[1] V. S. Lee, H. Rusinek, L. Bokacheva, A. Huang, N. Oesingmann, Q. Chen, M. Kaur, K. Prince, T. Song, E. Kramer, and E. Leonard, "Renal function measurements from MR renography and a simplified multicompartmental model," Am J Physiol Renal Physiol, vol. 292, pp. F1548-F1559, 2007

[2] S. E. Yuksel, A. El-Baz, A.A.Farag, M. E. A. El-Ghar, T.A.Eldiasty, and M.A.Ghoneim, "Automatic detection of renal rejection after kidney transplantation," International Congress Series vol. 1281, pp. 773-778, 2005.

[3] J. D. Grattan-Smith, M. R. Perez-Bayfield, R. A. Jones, S. Little, B. Broecker, E. A. Smith, H. C. Scherz, and A. J. Kirsch, "MR imaging of kidneys functional evaluation using F-15 perfusion imaging," Pediatric Radiology vol. 33, pp. 293-304, 2003.

[4] H. Rusinek, Y. Boykov, M. Kaur, S. Wong, L. Bokacheva, J. B. Sajous, A. J. Huang, S. Heller, and V. S. Lee, "Performance of an automated segmentation algorithm for 3D MR renography," Magn Reson Med, vol. 57, pp. 1159-1167, 2007.

[5] Y. Sun, J. M. F. Moura, D. Yang, Q. Ye, and C. Ho, "Kidney segmentation in MRI sequences using temporal dynamics," in IEEE International Symposium on Biomedical Imaging, 2002, pp. 98-101.

[6] Y. Boykov, V. S. Lee, H. Rusinek, and R. Bansal, "Segmentation of dynamic N-D data sets via graph cuts using markov models," in Proceedings of the 4th International Conference on Medical Image Computing and ComputerAssisted Intervention, 2001, pp. 1058-1066.

[7] J. A. d. Priester, A. G. Kessels, E. L. Giele, J. A. d. Boer, M. H. Christiaans, A. Hasman, and J. M. v. Engelshoven, "MR renography by semiautomated image analysis: performance in renal transplant recipients," J Magn Reson Imag, vol. 14, pp. 134-140, 2001.

[8] Y. Sun, M.-P. Jolly, and J. M. F. Moura, "Integrated Registration of Dynamic Renal Perfusion MR Images," in IEEE International Symposium on Image Processing, ICIP'04, Singapore, 2004.

[9] Q. Zhang, R. Souvenir, and R. Pless, "On Manifold Structure of Cardiac MRI Data: Application to Segmentation," IEEE Transaction on Medical Imaging, vol. 1, pp. 1092-1098, 2006.

[10] T. F. Chan and L. A. Vese, "Active contours without edges," IEEE Transactions on Image Processing, vol. 10, pp. 266 277, 2001.

[11] S. Osher and J. A. Sethian, "Fronts propagating with curvature-dependent speed: Algorithms based on HamiltonJacobi formulations," Journal of Computational Physics, vol. 79, pp. 12-49, 1988. 